\title{
Interpretación judicial, ambiente y dignidad
}

\author{
Judicial Interpretation, Environment and Dignity
}

Eduardo Esteban Magoja ${ }^{1}$

ORCID: 0000-0003-3182-5219

${ }^{1}$ Universidad de Buenos Aires, Argentina

Correo: magojaeduardo@gmail.com

Recibido: 19/09/2019

Aceptado: 17/02/2020

\section{Resumen:}

En el marco del desarrollo del denominado "Estado ambiental de derecho" existe un aspecto interesante a nivel iusfilosófico que tiene lugar directamente sobre la base de los compromisos asumidos por los jueces: el valor de la dignidad humana en relación con el cuidado del ambiente. Este trabajo se propone demostrar cómo en la jurisprudencia de los últimos años de la Corte Suprema de Justicia de la Nación argentina, concerniente al derecho al agua potable y la protección de los recursos hídricos, el respeto de la dignidad se encuentra en el centro de la labor interpretativa y constituye un criterio rector en la toma de decisiones judiciales.

Palabras clave: dignidad; interpretación judicial; ambiente; jurisprudencia; recursos hídricos.

\begin{abstract}
:
In the development of the so-called "Environmental Rule of Law", there is a very interesting feature which takes place directly based on the commitments assumed by judges: the value of human dignity in relation to the care of the environment. In relation to this, the propose of this paper is to demonstrate how in the recent case-law of the Supreme Court of Argentina, which is related to the human right to water and the protection of the hydrological resources, dignity is at the center of interpretive work and it also constitutes a guiding criterion in judicial decisionmaking.
\end{abstract}

Keywords: dignity; judicial interpretation; environment; jurisprudence; water resources. 


\section{Introducción}

En materia ambiental, la segunda mitad del siglo $\mathrm{XX}$ se caracterizó por la aparición de varias disciplinas, como por ejemplo la ecofilosofía, el derecho ambiental, la ética ambiental y el estudio de los "derechos de los animales" (Kawall, 2017, pp. 1223). En la actualidad, el desarrollo de estos saberes ayudó a establecer una mirada diferente acerca de la relación del hombre con la naturaleza y contribuyó a establecer las condiciones de posibilidad para la emergencia de una nueva forma de organización política: el "Estado ambiental de derecho", término que por cierto fue acuñado por primera vez en la doctrina alemana y fue introducido en Argentina por Quiroga Lavié (1996) bajo el rótulo de "Estado ecológico de derecho" (pp. 950-960).

Este escenario presenta características novedosas en el campo jurídico (Esain, 2017; Comastri y Villalba, 2018; Lubertino Beltrán, 2018; Pellegrini, 2018). Así, en relación con la labor judicial, Falbo (2019a) destaca que la Corte Suprema de Justicia de la Nación argentina (en adelante CSJN) "ha venido elaborando una jurisprudencia de tutela y protección ambiental no solo de innegable excelencia e importancia mayúscula, sino a la vez de vanguardia” (p. 1). En este sentido, algunos de los aspectos más sobresalientes son: la exégesis amplia de las reglas procesales en los asuntos relativos al daño ambiental, la presencia de una perspectiva ecocéntrica, la necesidad de jueces activos en lugar de meros "jueces espectadores", la prioridad absoluta en la prevención del daño futuro y la existencia de principios especiales que informan la materia, tales como el principio precautorio, de prevención, de progresividad y de participación ciudadana entre otros. ${ }^{1}$ Sin embargo, en relación con la base de los compromisos asumidos por los jueces, existe un aspecto iusfilosófico mucho más interesante, cuyo estudio ha sido un poco descuidado en la literatura: el valor y el trato privilegiado que recibe la dignidad humana en relación con el cuidado ambiental.

\footnotetext{
${ }^{1}$ Además de en los fallos de la CSJN que serán objeto de estudio en este trabajo, tales aspectos se pueden observar también en "Mendoza Beatriz Silva y otros c/Estado Nacional" (2006, 2007, 2008, 2015 y 2018), "Salas, Dino y otros c/Salta, Provincia de y Estado Nacional” (2008 y 2009), "Cruz, Felipa y otros c/Minería Alumbrera Limited y otro" (2016), "Martínez, Sergio Raúl c/Agua Rica LLc Suc. Argentina y su propietaria Yamana Gold Inc. y otro" (2016), "Asociación Argentina de Abogados Ambientalistas de la Patagonia c/Santa Cruz, Provincia de y otro" (2016), "Fundación Ciudadanos Independientes c/San Juan, Provincia de, Estado Nacional y otros" (2016), y "Mamani, Agustín Pio y otros c/Estado Provincial -Dirección Provincial de Políticas Ambientales y Recursos Naturales- y la Empresa Cram S.A.” (2017).
} 
Mediante una metodología convencional para investigaciones en la que se entrecruzan cuestiones iusfilosóficas y estudios de fallos, este trabajo se propone demostrar cómo en la reciente jurisprudencia de la CSJN, referente al derecho al agua potable y la protección de los recursos hídricos, el respeto de la dignidad humana se encuentra en el centro de la labor interpretativa y constituye un criterio rector en la toma de decisiones judiciales. A tales fines, el objeto de estudio serán los siguientes fallos: "Kersich, Juan Gabriel y otros c/Aguas Bonaerenses S.A.” (2014); "La Pampa, Provincia de c/Mendoza, Provincia de" (2017); "Barrick Exploraciones Argentinas S.A. y otro c/Estado Nacional” (2019); y "Majul, Julio Jesús c/Municipalidad de Pueblo General Belgrano y otros" (2019). Vale aclarar que esta selección no es arbitraria, sino que se justifica debido a que tales fallos son los más actuales en la materia y aquellos en donde se han abordado los temas de nuestro interés con mayor detalle y profundidad.

En lo que a la organización del artículo se refiere, se sigue el siguiente orden expositivo: en primer lugar, se desarrollará el concepto de dignidad desde una mirada filosófica y se explicará, a su vez, cómo es el elemento fundacional de los derechos humanos; en segundo término, se examinará su reconocimiento en la normativa internacional y local; estas consideraciones permitirán abordar, en tercer lugar, su recepción y el carácter dado en los fallos indicados; finalmente, se realizarán unas breves conclusiones.

\section{El concepto de dignidad humana}

El primer paso de este trabajo, como se ha dicho, es desarrollar el concepto de dignidad y explicar cómo es posible pensar que ella opera como fundamento de los derechos humanos. Esto permitirá ver luego el papel que cumple en la labor interpretativa de los jueces. Vale aclarar que no interesa realizar un análisis histórico ni tampoco estudiar en profundidad la temática. Semejante empresa sería imposible si se considera la abundante literatura existente. ${ }^{2}$ El objetivo es más modesto y se centra en realizar unas consideraciones básicas del concepto bajo una perspectiva de índole filosófica.

\footnotetext{
${ }^{2}$ Así, por ejemplo, Pritchard (1972), Gaylin (1984), González Pérez (1986), Spaemann, (1988), Arendt (1992), Bristow (1993), Melendo y Millán-Puelles (1996), Andorno (1997), D’Agostino (2002), Mattson y Clark (2011), Kateb (2011), Ober (2012), Michael (2014), Millán-Puelles (2014) y Massini Correas (2017), entre muchos otros.
} 
En tal sentido, una primera observación, que ayuda a entrar en el tema, es que se pueden identificar, siguiendo a Michael (2014), dos categorías de dignidad: una "dignidad inherente" (inherent dignity) y una "dignidad no inherente" (non-inherent dignity). Mientras que la primera hace referencia a un valor que le pertenece por igual a todos los seres humanos y se caracteriza por ser incondicionado, indivisible, permanente e inviolable, la segunda indica una cualidad adquirida que es variable, contingente, admite grados de desarrollo y está determinada en base a criterios culturales acerca de lo que es digno. Por cierto, entre ambas categorías existe un orden de prioridad. En efecto, como explica Laise (2017):

la dignidad inherente se antepone a la no inherente o, lo que es lo mismo, la idea de que la dignidad se va actualizando o realizando en las personas parte necesariamente del reconocimiento de un valor intrínseco, innato, indisponible, no instrumentalizable de todo individuo (p. 134).

De esta distinción, también expresada por algunos como la diferencia entre “dignidad ontológica” y “dignidad adquirida” (Millán-Puelles, 2014), en lo que sigue se analizará en mayor medida la dignidad inherente, dado su carácter primario. Semejante postura se remonta a Kant (Hervada, 2000, p. 447), cuyas consideraciones, además de imprescindibles, son la guía más clara para transitar el complejo y sinuoso camino que involucra la comprensión del concepto. En efecto, según el filósofo de Königsberg, el concepto se relaciona con la idea de que los seres racionales tienen un valor intrínseco (Caimi, 2017, p. 142). La dignidad (Würde) es la propiedad de estar por encima de todo precio y, en consecuencia, de no tener equivalente ni nada que lo reemplace (Kant, 2008, pp. 123-24); por esta razón, se dice que el hombre es un fin en sí mismo (Kant, 2016, p. 403). Incluso, dice Kant (2008), la autonomía, en el sentido de autolegislación moral, es el fundamento de la naturaleza humana y de toda naturaleza racional (p. 125).

Tales ideas presentan a su vez un fuerte vínculo con el concepto de respeto. Así pues, Tugendhat (1992) dice que cuando "respetamos a una persona no por algo, sino simplemente como persona, la respetamos como ser autónomo y podemos decir que al hacerlo lo que respetamos es precisamente la dignidad de la persona" (p. 251). Sin embargo, la cuestión no termina allí. Al proceder de ese modo, se reconoce a la persona como sujeto de derecho, lo cual es palpable en la expresión "respeto de sí mismo". En efecto, tener respeto de sí mismo implica que uno tiene consciencia de ser autónomo y 
además titular de derechos iguales a los de todos los demás miembros de la comunidad. No está de más decir que la dignidad no es un concepto que expresa simplemente una cualidad inherente a una persona, considerada como un ser aislado y abstraído del mundo y las relaciones humanas. Como explica Tugendhat (1992), la dignidad debe ser pensada como "algo que es aceptado cuando hay respeto mutuo" (p. 252). Ella siempre implica a otro. Así, en tanto el Estado tenga basamento en la dignidad de la persona necesariamente se concibe "como constituido por ciudadanos que tienen respeto mutuo por la autonomía de cada uno de los demás" (Tugendhat,1992, p. 252).

La idea del respeto mutuo se conecta con otro punto que comprende el concepto de dignidad: los deberes. En efecto, Quintana (2017) sostiene que, desde una perspectiva que revalorice la relación derechos/deberes, la dignidad adquiere toda su trascendencia jurídica al asumirse tanto en su sentido ontológico como desde

su rol activo o moral, lo que significa que toda persona tiene la obligación o el deber de comportarse conforme su constitutivo esencial humano para alcanzar la dignidad de su naturaleza, tanto en sí misma como frente a los demás (p. 85).

Bajo tal perspectiva, pues, se puede decir que la dignidad humana involucra deberes en una doble dirección: deberes personales, pero también hacia el resto de los seres humanos. Vivir con dignidad es respetarse tanto a sí mismo como a los demás, no instrumentalizarse ni instrumentalizar a los otros: para decirlo en pocas palabras, no es otra cosa que tratarse a sí mismo y a todo hombre como fin y no como simple medio. Entonces, la dignidad en tanto relación, depende del reconocimiento del valor de los otros y del trato que ellos se merecen en virtud de ser personas. Quien se comporte de este modo, de hecho, demuestra su propia dignidad (Dupré, 2013).

Existe una estrecha conexión entre dignidad, igualdad, respeto mutuo y autonomía. En especial, es con este último concepto con el cual se suele vincular la dignidad, tal como vimos con Kant. En esta misma dirección, aunque desde una perspectiva filosófica distinta, se sitúa Dworkin (2006), quien dice que las dos dimensiones de la dignidad son: primero, que cada vida humana tiene un valor potencial intrínseco y, segundo, que todos tienen la responsabilidad de realizar, mediante sus elecciones autónomas, ese valor en su propia vida (p. 10). 
Quien ingeniosamente articula la dignidad con los términos mencionados es Alexy (2007), precisamente cuando indaga el problema de la fundamentación de los derechos humanos $^{3}$ y propone el reconocimiento del hombre en tanto "criatura discursiva", autónoma, libre, igual y con valor en sí mismo. En efecto, al momento en que Alexy brinda una fundamentación explicativa de tales derechos, dice que ello se logra demostrando que la praxis del afirmar (Behaupten), del preguntar (Fragen) y del aducir (Anführen) presupone la libertad y la igualdad, que los seres humanos son autónomos y que tienen una naturaleza discursiva. El hombre, al hacer uso de sus competencias discursivas, reconoce al otro como participante del discurso con igualdad de derechos. Si, además, se conduce con seriedad, lo reconoce también como autónomo; este reconocimiento, pues, supone a su vez aceptar al otro como persona que tiene valor y dignidad. Sin embargo, para Alexy (2007) el argumento explicativo es insuficiente ${ }^{4}$ y hay que complementarlo con un argumento existencial, según el cual la cuestión gira en torno a si nosotros mismo nos vemos como criaturas discursivas (p. 244). Se trata, ni más ni menos, que de una decisión acerca de lo que nosotros somos. Aunque el autor no lo diga de este modo, lo que deja entrever este segundo argumento es que, en la práctica discursiva, no se cancela la fundamentación ontológica y se vuelve a definir al hombre a la vieja manera en que lo hizo Aristóteles en Política (1253a9-10), es decir, como "animal dotado de lógos".

Queda claro, en función de lo expuesto, cómo la dignidad constituye un concepto que expresa el valor intrínseco de las personas. Esto lo convierte en un principio básico y apropiado sobre el cual apoyar toda argumentación referente a los derechos humanos. En relación con este aspecto, Spaemann (1988) sostiene que el concepto de dignidad humana

\footnotetext{
${ }^{3}$ El problema de la fundamentabilidad se puede sintetizar en la cuestión acerca de cómo es posible justificar "las normas o reglas morales que con pretensión de prioridad garantizan los derechos universales, fundamentales y abstractos" (Alexy, 2007, p. 239). Dejando de lado los enfoques que niegan la posibilidad de tal fundamentabilidad (los enfoques escépticos), Alexy (2007) admite que esto es posible realizar prima facie desde ocho posturas no-escépticas: la religiosa, la biológica, la intuitiva, la consensual, la instrumental, la cultural, la explicativa y la existencial. Sin embargo, ninguna de las seis primeras propuestas logra dar una respuesta satisfactoria a la cuestión. Los derechos humanos solo se pueden fundamentar a partir de una combinación de características explicativas y existenciales, que es la postura por la cual se inclina el profesor de Kiel. Sobre la cuestión de la fundamentación de los derechos humanos ver Alexy (1996, 2007, 2013).

${ }^{4}$ El argumento es insuficiente porque falla, por lo menos, en dos puntos. En primer lugar, se puede eludir la necesidad de las reglas del discurso al evitar toda participación en la práctica del afirmar, preguntar y discutir. El precio de esto es muy alto, ya que el hombre como "criatura discursiva" debería renunciar a aquello que lo caracteriza. El segundo problema proviene de la diferencia entre, por un lado, discurso y acción, y por el otro, entre las capacidades y los intereses. Que se tengan capacidades discursivas no implica un interés en hacer uso de ella (Alexy, 2007, p. 244).
} 
no se refiere a un derecho en especial, sino que "contiene la fundamentación de lo que puede ser considerado como Derecho Humano en general. Lo que con él se nombra es algo más originario de lo que se expresa por medio del término 'Derecho Humano"' (p. 15). También, de un modo semejante, Massini Correas (1994) afirma que "sin la idea de la dignidad de la persona humana es inconcebible la noción misma de derechos humanos" (p. 136).

Bajo esta perspectiva, se puede decir que la dignidad tiene un carácter axiomático. De hecho, esta lectura se condice con la semántica del vocablo latino dignitas, del cual deriva nuestro término actual "dignidad", y también de la palabra griega "axioma" $(\dot{\alpha} \xi \hat{\xi} \omega \mu \alpha)$. En efecto, dignitas (sobre todo su forma plural dignitates) y $\dot{\alpha} \xi i ́ \omega \mu \alpha$ no solo aluden a una cualidad humana (como el honor, la reputación o el rango), sino que también hacen referencia a las "proposiciones evidentes", los "principios indemostrables" que son asumidos como la base mediante la cual se construye una demostración (Magnavacca, 2014, p. 215; Glare, 1968, p. 542; Liddell y Scott, 1996, p. 172). Tal como sostiene Millán-Puelles (2014), “axiómata, dignitates, son, en el orden lógico — no en el psicológico-, las verdades objetivamente irreductibles, las que valen en sí, sin posibilidad de mediación" (p. 84). Entonces, pensado en estos términos, la dignidad es un concepto valioso que nos sirve como principio y punto de partida para construir todo la estructura política y jurídica, la cual debe estar al servicio del hombre y establecer las condiciones necesarias que permitan su desarrollo de acuerdo con el plan de vida elegido. Sin ir más lejos, en el fallo "Aquino, Isacio c/Cargo Servicios Industriales S.A.” (2004), la CSJN sostuvo que el fundamento definitivo de los derechos humanos, enunciado desde hace más de medio siglo por la Declaración Universal de Derechos Humanos de 1948, es "la dignidad del ser humano, que no deriva de un reconocimiento ni de un gracia de las autoridades o poderes, toda vez que resulta 'intrínseca' o 'inherente' a todas y cada una de las personas humanas y por el solo hecho de serlo".

\section{La recepción de la dignidad en la normativa internacional y local}

La exposición acerca de la dignidad, que ha sido hasta aquí de índole filosófica, no estaría completa si no se expone por lo menos cómo se la entiende en el ámbito jurídico-normativo. Lo interesante de esta dimensión es que respalda precisamente la tesis que se viene sostenido aquí acerca de que la dignidad es el fundamento de los derechos. En efecto, en el plano internacional, la Declaración Universal de Derechos Humanos de 
1948 establece, en su primer considerando, que "la libertad, la justicia y la paz en el mundo tienen por base el reconocimiento de la dignidad intrínseca y de los derechos iguales e inalienables de todos los miembros de la familia humana”. Más adelante, en el quinto considerando, declara que "los pueblos de las Naciones Unidas han reafirmado en la Carta su fe en los derechos fundamentales del hombre, en la dignidad y el valor de la persona humana y en la igualdad de derechos de hombres y mujeres, y se han declarado resueltos a promover el progreso social y a elevar el nivel de vida dentro de un concepto más amplio de la libertad". En su artículo $1^{\circ}$ reconoce que "todos los seres humanos nacen libres e iguales en dignidad y derechos y, dotados como están de razón y conciencia, deben comportarse fraternalmente los unos con los otros".

En la misma línea que la Declaración e incluso utilizando casi las mismas palabras, tanto el Pacto Internacional de Derechos Económicos, Sociales y Culturales como el Pacto Internacional de Derechos Civiles y Políticos, ambos de 1966, dicen que "la libertad, la justicia y la paz en el mundo tienen por base el reconocimiento de la dignidad inherente a todos los miembros de la familia humana y de sus derechos iguales e inalienables". Incluso, agregan un elemento de especial interés. En el segundo considerando aclaran expresamente que "estos derechos se desprenden de la dignidad inherente a la persona humana".

En el ámbito regional, la Convención Americana sobre Derechos Humanos de 1969 hace referencia en varias ocasiones, directa o indirectamente, a la dignidad. Así, por ejemplo, en el preámbulo sostiene que "los derechos esenciales del hombre no nacen del hecho de ser nacional de determinado Estado, sino que tienen como fundamento los atributos de la persona humana", y en su artículo $11^{\circ}$ establece que "toda persona tiene derecho al respeto de su honra y al reconocimiento de su dignidad".

En lo que respecta al plano del derecho interno, varios países, como Alemania y España, reconocen la importancia de la dignidad humana. ${ }^{5}$ En cuanto a nuestro país, entre las diferentes leyes que la mencionan, se destaca el artículo $51^{\circ}$ del Código Civil y

\footnotetext{
${ }^{5}$ Así, la Ley Fundamental de Alemania de 1949, en su artículo $1^{\circ}$ inciso $1^{\circ}$ sostiene que "la dignidad humana es intangible. Respetarla y protegerla es obligación de todo poder público", y en el inciso $2^{\circ}$ agrega que "el pueblo alemán, por ello, reconoce los derechos humanos inviolables e inalienables como fundamento de toda comunidad humana, de la paz y de la justicia en el mundo". En lo que respecta a la Constitución Española de 1978, el artículo $10^{\circ}$ inciso $1^{\circ}$ dice que "la dignidad de la persona, los derechos inviolables que le son inherentes, el libre desarrollo de la personalidad, el respeto a la ley y a los derechos de los demás son fundamento del orden político y de la paz social", y también en su preámbulo se declara el compromiso de la Nación española en "promover el progreso de la cultura y de la economía para asegurar a todos una digna calidad de vida".
} 
Comercial de 2015, el cual dice que "la persona humana es inviolable y en cualquier circunstancia tiene derecho al reconocimiento y respeto de su dignidad". ${ }^{6}$ Sin embargo, en comparación con la mayoría de los instrumentos internacionales mencionados, se advierte aquí una diferencia fundamental acerca de cómo la dignidad se concibe. En efecto, no se hace referencia a ella como aquello que proporciona las bases para un conjunto básico de derechos humanos, sino que se la entiende, de modo semejante al artículo $11^{\circ}$ de la Convención Americana sobre Derechos Humanos, en el sentido de que las personas deben ser tratadas con respeto o, lo que es lo mismo, de que tienen derecho a ser tratadas con dignidad. El "derecho a la dignidad" o el "derecho al reconocimiento de la dignidad" significa, para ser más precisos, que la dignidad misma es objeto de tutela jurídica: en palabras de Michael (2014), "un fin deseado que los derechos concretos deben proteger" (p. 12).

La cuestión acerca de si la dignidad opera como fundamento de los derechos o, en su lugar, se trata de un objeto privilegiado que protegen los derechos es un tema controvertido. Se puede llegar a pensar, incluso, que se trata de dos concepciones incompatibles. No obstante, no hace falta llegar a semejante extremo y que es posible encontrar, más que exclusión, una conciliación entre ambas lecturas. En efecto, la dignidad, de acuerdo con la categorización de Michael (2014), no solo abarca una dimensión vinculada al valor inherente de la persona, sino que ella, en su perfil "no inherente", comprende aquello que deseamos adquirir en nuestra vida moral dentro de un contexto histórico y cultural determinado. En la vida moral desarrollamos nuestra dignidad bajo la perspectiva de lo que consideramos y se considera "digno" y tal comportamiento se vincula por lo general con aquello valioso para garantizar la dignidad inherente. Entonces, bajo esta manera de ver las cosas, es natural desear que la dignidad que se materializa en el despliegue del plan de vida elegido tenga tutela jurídica bajo el ropaje de un derecho. Hablar de derecho a la dignidad, pues, no cancela ni crea confusión en la idea de considerarla como fundamento de los derechos. Se trata de una perspectiva complementaria, que ve la dignidad desde otro ángulo.

\footnotetext{
${ }^{6}$ En relación con el concepto de dignidad en el artículo $51^{\circ}$ del Código Civil y Comercial de 2015, ver en especial el trabajo de Consolo (2017).
} 


\section{La dignidad en el centro de la interpretación judicial}

Se ha llegado al punto de volcar las consideraciones teorías realizadas al plano práctico de la labor interpretativa judicial de la CSJN. Se tratará de demostrar cómo la dignidad, entendida como pilar básico de la arquitectura jurídico-política y a la cual el Estado debe enfocar todos sus esfuerzos para protegerla, aparece reconocida en los fallos “Kersich” (2014), "La Pampa” (2017), "Barrick” (2019) y "Majul” (2019). Es más, se verá que los jueces, ya sea implícita o explícitamente, se valen de ella como principio rector en su labor interpretativa. Sin entrar a describir en detalle los hechos de los casos - algo que, además de innecesario, se puede suplir con su simple lectura - en lo que sigue se expondrán los argumentos mediante los cuales los miembros de la CSJN sostienen aquella postura en relación con el problema del derecho al agua potable y la protección de los recursos hídricos. ${ }^{7}$

En relación con el fallo "Kersich" (2014), lo primero que habría que señalar es que los jueces abordaron el derecho humano al agua y su especial carácter de derecho de incidencia colectiva; asimismo, destacaron su vínculo directo sobre la vida y la salud de las personas como así también que es una cuestión que excede el mero interés individual. En sentido, recordaron la resolución $27 / 7$ aprobada el 2 de octubre de 2014 en la $27^{\circ}$ sesión del Consejo de Derechos Humanos de la Asamblea General de Naciones Unidas, en la que se exhorta a los Estados a que "velen por que todas las personas tengan acceso sin discriminación a recursos efectivos en caso de violación de sus obligaciones respecto del derecho humano al agua potable y el saneamiento, incluido recursos judiciales, cuasijudiciales y otros recursos apropiados".

En la misma línea, señalaron la tutela que tiene semejante derecho en los instrumentos internacionales. Así por ejemplo, se hizo mención: a la resolución 64/292, dictada el 30 de julio de 2010 por la Asamblea General de Naciones Unidas, que "declaró el derecho al agua potable y el saneamiento como derecho humano esencial para el pleno disfrute de la vida y de todos los derechos humanos"; al artículo $14^{\circ}$ párrafo $2^{\circ}$ de la Convención sobre la Eliminación de todas las Formas de Discriminación contra la Mujer de 1979 y al denominado "Protocolo de San Salvador" de 1988, que sostienen que "toda

\footnotetext{
${ }^{7}$ En función de los propósitos fijados, no se analizarán todos los aspectos de los fallos. A tales fines, se pueden consultar los comentarios que se realizan en los trabajos de Sabsay y Fernández (2019), Falbo (2019b), Esain (2018, 2019), Catalano (2019), Pinto y Liber (2015), Rodríguez (2019).
} 
persona tiene derecho a un medio ambiente sano y a contar con los servicios básicos"; a la Convención sobre los Derechos del Niño de 1989, que en su artículo $24^{\circ}$ párrafo $2^{\circ}$ exige a los Estados que luchen contra las enfermedades mediante el suministro de agua potable salubre. También los integrantes de la CSJN destacaron cómo el derecho se encuentra reconocido en numerosos documentos de organismos internacionales, de los cuales citaron la Observación General 15 del Comité de Derechos Económicos, Sociales y Culturales de Naciones Unidas, que en el 2002 declaró que "el agua es un recurso natural limitado y un bien público fundamental para la vida y la salud. El derecho humano al agua es indispensable para vivir dignamente y es condición previa para la realización de otros derechos humanos [cursivas añadidas]".

En lo que respecta al fallo "La Pampa" (2017), los jueces se apoyaron en la doctrina fijada en el fallo "Kersich" (2014) e insistieron sobre la idea de que "es fundamental la protección del agua para que la naturaleza mantenga su funcionamiento como sistema y su capacidad regenerativa y de resiliencia". También, en base a los lineamientos trazados en diversos instrumentos internacionales, muchos de los cuales fueron mencionados en el fallo "Kersich" (2014) y que no hace falta volver a nombrar, se caracterizó el derecho al acceso al agua potable como derecho humano fundamental y necesario para el desarrollo de la persona. En relación con este ámbito, es importante señalar dos aspectos que la CSJN enfatiza. En primer lugar, que de las resoluciones de la Asamblea General de Naciones Unidas "se dice que los Estados deben adoptar estrategias y programas amplios e integrados para velar porque las generaciones presentes y futuras dispongan de agua suficiente y salubre". El segundo punto que es de interés, es la remisión a la jurisprudencia de la Corte Interamericana de Derechos Humanos que ha estudiado la materia. Así, en el caso "Comunidad indígena Yakye Axa vs. Paraguay” del 17 de junio de 2005, señaló que "las afectaciones [...] del derecho al agua limpia impactan de manera aguda el derecho a una existencia [...], para el ejercicio de otros derechos" y, en el caso "Vélez Loor vs. Panamá" del 23 de noviembre de 2010, dijo que "el derecho al agua potable y el saneamiento es un derecho humano esencial para el pleno disfrute de la vida".

En el fallo "Barrick" (2019) la CSJN volvió a hacer uso de la mayoría de los argumentos expuestos en sus precedentes. Sin embargo, ofrecieron un agregado teórico importante, que se vincula al tema conflictivo que tuvieron que resolver: determinar la constitucionalidad de la ley 26.639 acerca de los "Presupuestos Mínimos para la Preservación de los Glaciares y del Ambiente Periglacial”. Así, en relación con la 
necesidad de proteger los glaciares y periglaciares argentinos, destacó su función de reservas estratégicas de recursos hídricos para el consumo humano y el respeto de la biodiversidad, e hizo también hincapié en cómo dicha ley afronta una nueva problemática ambiental que debe ser considerara con prudencia y sin perderse de vista que el ambiente es un bien colectivo, de pertenencia comunitaria, de uso común e indivisible, el cual debe ser preservado no solo en función de los interés actuales, sino con responsabilidad hacia las generaciones futuras. Se trata, como dice la CSJN en una formulación central de la sentencia, de tomar "los derechos colectivos ambientales... en serio", pues "su operatividad abre novedosos ámbitos de deliberación política y responsabilidad jurídica insospechada pocas décadas atrás". ${ }^{8}$

Finalmente, en el caso "Majul” (2019) la CSJN dictó una sentencia histórica en cuanto a la protección de los humedales. Mediante su decisión se dejó sin efecto la sentencia del Superior Tribunal de Justicia de la Provincia de Entre Ríos que había rechazado una acción de amparo interpuesta con el objeto de que cesen las obras y se reparen los perjuicios ambientales producidos por el proyecto inmobiliario "Amarras de Gualeguaychú".

Al respecto, destacó que se llevaron a cabo acciones para la construcción del barrio que dañaron el ambiente y que en virtud de su gravedad y magnitud podrían ser de difícil e, incluso, imposible reparación ulterior. En este sentido, sostuvo que el Superior Tribunal de Entre Ríos “omitió considerar normas tendientes a demostrar que la acción de amparo era la vía adecuada para la tutela de los derechos invocados". También agregó que dicho Tribunal no tuvo en cuenta el derecho a vivir en un ambiente sano, conforme lo disponen los artículos $41^{\circ}$ de la Constitución Nacional y $22^{\circ}$ de la Constitución de Entre Ríos, ni tampoco que el gobierno local es responsable por la gestión y el uso sustentable de las cuencas hídricas y "los sistemas de humedales que se declaran libres de construcción de obras de infraestructura a gran escala que puedan interrumpir o degradar la libertad de sus aguas y el desarrollo natural de sus ecosistemas asociados", tal como lo establece el artículo $85^{\circ}$ de la Constitución provincial.

Tras destacar el valor natural de los humedales y la necesidad de proceder a su protección en virtud de la afectación que generaría en los derechos de los habitantes y del

\footnotetext{
${ }^{8}$ Así, pues, como señala Falbo (2019a), "tomar en serio los derechos colectivos ambientales no es una posición meramente teórica o doctrinaria, sino también, simultáneamente, un compromiso de la justicia con la eficacia operativa del derecho ambiental" (p. 1-9).
} 
impacto irreversible que significaría para el ecosistema, los jueces introdujeron el aspecto más novedoso del fallo. Afirmaron que el Estado no solo debe garantizar la aplicación de los principios de sustentabilidad, precaución, equidad intergeneracional, prevención, utilización racional, progresividad y responsabilidad, sino que en cuestiones en las que están en juego los recursos hídricos corresponde valorar dos principios más: el principio in dubio pro natura, el cual establece que, en caso de duda, las decisiones deberán adoptarse en favor de la protección y conservación del ambiente, ${ }^{9}$ y el principio in dubio pro aqua que dispone la interpretación de leyes "del modo más favorable a la protección y preservación de los recursos de agua y ecosistemas conexos".

Ahora bien, lo que demuestran los fallos "Kersich" (2014), "La Pampa" (2017), "Barrick" (2019) y "Majul" (2019) es que el Estado debe garantizar el acceso al agua potable y la protección de los recursos hídricos en virtud de la dignidad del hombre, ya que de otro modo no sería posible su desarrollo personal ni su existencia. Esta postura se puede apreciar, por lo menos, en tres facetas o dimensiones. En primer lugar, existen ciertas afirmaciones por parte de los jueces que van precisamente en esa dirección. Será suficiente con señalar tres: en primer lugar, cuando se sostiene que el derecho al agua es la condición de posibilidad de la existencia humana; en segundo término, al destacar que es un bien público fundamental para la salud que, incluso, resulta indispensable para vivir dignamente y disfrutar de los otros derechos humanos; finalmente, en relación con la protección de los humedales, cuando los jueces explican que la alteración del ecosistema afecta la biodiversidad y el sistema de la cuenca hidrográfica, lo cual repercute en un detrimento de los derechos de los habitantes de Entre Ríos.

La segunda dimensión se vincula con los criterios interpretativos asumidos. En efecto, los argumentos de los jueces de la CSJN, en la medida que colocan su atención directamente en garantizar un ambiente sano y equilibrado en el que el hombre pueda ejercer sus derechos y desarrollarse como persona, reconocen que solo bajo tales condiciones se le da un trato digno y se lo respeta en tanto criatura discursiva con un valor intrínseco, inviolable y permanente. Todos los esfuerzos interpretativos se dirigen hacia

\footnotetext{
${ }^{9}$ En relación con este principio invocado en la sentencia, Rodríguez (2019), en su comentario al fallo, considera que en realidad debería ser más amplio: el principio pro ambiente o in dubio pro vida.
} 
una misma dirección: el respeto de la dignidad humana y, en consecuencia, el de los derechos fundamentales del hombre en el marco de una perspectiva ecocéntrica. ${ }^{10}$

En efecto, la protección de los recursos hídricos, la conservación de los ecosistemas y el cuidado de las reservas hídricas buscan conservar la salud de la Tierra, hogar común y "madre bella que... acoge entre sus brazos" a los seres humanos y a todas las demás formas de vida (Encíclica papal Laudato si’, 2015, § 1). Sin condiciones naturales mínimas y apropiadas para la vida, estamos expuestos a sufrir un deterioro considerable en el disfrute de los derechos. Incluso, en situaciones extremas, la subsistencia de la humanidad corre peligro. La CSJN ha mostrado un fuerte compromiso en adoptar medidas efectivas conducentes a obtener "un ambiente sano, equilibrado, apto para el desarrollo humano y para que las actividades productivas satisfagan las necesidades presentes sin comprometer las de las generaciones futuras", tal como prescribe nuestra propia Constitución en su artículo $41^{\circ}$.

La última faceta es que el enfoque que privilegian los jueces acerca del respeto de la dignidad humana revela el lugar especial que recibe la persona, en tanto titular de derechos, dentro de los Estados actuales. En efecto, en un Estado democrático de derecho, que los individuos tengan derechos significa que ellos tienen no solo protección, sino también posibilidad de realizar su plan de vida, es decir, de ejercer su autonomía como seres independientes (Tugendhat, 1992, p. 252).

En tal sentido, Nino (1989) dice que el principio de autonomía de las personas "prescribe que siendo valiosa la libre elección individual de planes de vida y la adopción de ideales de excelencia humana, el Estado (y los demás individuos) no debe interferir en esa elección o adopción” (p. 204). Su ejercicio se pone en peligro por las interferencias que pueda realizar el Estado o un particular, de manera que el aparato estatal político debe limitarse —agrega Nino (1989)— “a diseñar instituciones que faciliten la persecución individual de esos planes de vida y la satisfacción de los ideales de virtud que cada uno sustente e impidiendo la interferencia mutua en el curso de tal persecución” (pp. 204-

\footnotetext{
${ }^{10}$ Así, en relación con este punto, en el fallo "La Pampa" la CSJN afirma que la "regulación jurídica del agua se ha basado en un modelo antropocéntrico, que ha sido puramente dominial al tener en cuenta la utilidad privada que una persona puede obtener de ella o bien en función de la utilidad pública identificada con el Estado [...] El paradigma jurídico que ordena la regulación del agua es eco-céntrico, o sistémico, y no tiene en cuenta solamente los intereses privados o estaduales, sino los del mismo sistema, como bien lo establece la ley general del ambiente. El ambiente no es para la Constitución Nacional un objeto destinado al exclusivo servicio del hombre, apropiable en función de sus necesidades y de la tecnología disponible, tal como aquello que responde a la voluntad de un sujeto que es su propietario". Esta cosmovisión, por cierto, se repite expresamente también en los fallos "Barrick" (2019) y "Majul" (2019).
} 
205). Sin embargo, no hay que perder de vista que la autonomía y, junto con ella, la dignidad, también se lesiona cuando el gobierno no garantiza condiciones favorables y mínimas que permitan desarrollar su plan de vida (Tugendhat, 1992, p. 252). La carencia de dichas condiciones, que abarca no solo cuestiones ambientales, sino también económicas y sociales, es un daño hacia la dignidad humana ${ }^{11} \mathrm{y}$, por extensión, un impedimento para el goce de los derechos del hombre ${ }^{12}$ y su propio desarrollo. ${ }^{13}$

\section{Conclusiones}

El desglose que se ha realizado aquí permite dar cuenta del lugar que ocupa en los fallos de la CSJN la persona como criatura discursiva con un valor intrínseco, cuyo fundamento último es la autonomía. En materia ambiental en general y en relación con el derecho al acceso al agua en particular, el Estado debe garantizar la capacidad que tiene la persona para llevar a cabo el curso de sus acciones en un ambiente sano, equilibrado y en el cual sea posible su desarrollo. Sin duda, el autogobierno de la persona, es decir, la facultad humana de ser autónomo, exige la ausencia de limitaciones significativas que la anulen por completo o, lo que es lo mismo, que el hombre tenga condiciones fácticas y jurídicas mínimas que le permitan llevar a cabo su plan vida. El acceso al agua potable y un ambiente adecuado son sin duda una de tales condiciones, tal como lo ha reconocido la CSJN en su reciente jurisprudencia.

En los tiempos actuales, la cuestión ambiental se ha vuelto uno de los temas que ningún Estado puede desconocer. En vistas a preservar los recursos de la naturaleza, se requiere, además de la participación de todos, decisiones públicas apropiadas que se focalicen en distintos puntos de acción. En este sentido y bajo la premisa de promover el desarrollo sostenible, la CSJN ha dirigido sus esfuerzos a encaran los conflictos desde una perspectiva que no descuida las múltiples facetas involucradas y que tiene como dirección privilegiada el respeto de la dignidad y los derechos fundamentales del hombre.

\footnotetext{
${ }^{11}$ Así, en términos de Alexy (2014) desde el punto de vista del derecho la protección de la dignidad no se restringe a la autolegislación moral, sino que "incluye, por ejemplo, el derecho a existir y el derecho a efectuar elecciones de cualquier clase" (p. 18).

12 De hecho, en este sentido, Lorenzetti (2015) advierte, al analizar el Código Civil y Comercial, que en "materia medioambiental se ha sostenido que la preservación de este macro bien opera como una suerte de presupuesto o prerrequisito para el disfrute de los demás derechos, motivo por el cual, en casos de colisión de normas tuitivas, debe estarse siempre a privilegiar la protección del ambiente" (p. 18).

${ }^{13}$ Sobre el desarrollo humano como paradigma constitucional, ver López Alfonsín (2012, pp. 19-28).
} 


\section{Referencias}

Alexy, R. (1996). Discourse Theory and Human Rights. Ratio Juris, 9(3), 209-235.

Alexy, R. (2007). ¿Derechos humanos sin metafísica? Doxa, 30, 237-248.

Alexy, R. (2013). Derecho, moral y existencia de los derechos humanos. Signos Filosóficos, 15(30), 153-171.

Alexy, R. (2014). La dignidad humana y el juicio de proporcionalidad. Parlamento y Constitución. Anuario, 16, 9-27.

Andorno, R. (1997). La bioétique et la dignité de la personne. París, Francia: PUF.

Arendt, H. (1992). La condición humana. Barcelona, España: Paidós.

Bristow, P. (1993). The Moral Dignity of Man. Dublín, Irlanda: Four Courts Press.

Caimi, M. (Coord). (2017). Diccionario de filosofía crítica kantiana. Buenos Aires, Argentina: Colihue.

Catalano, M. (2019). El fallo "Barrick” en clave constitucional. La Ley, Tomo 2019-D, 378-380.

Código Civil y Comercial de la Nación. (2015). Recuperado de http://servicios.infoleg.gob.ar/infolegInternet/anexos/235000239999/235975/norma.htm

Comastri, D., y Villalba, M. E. (2018). Hacia la consolidación del componente ambiental del Estado de Derecho: análisis de conflictos ambientales de la Provincia de Córdoba. Revista de Derecho Ambiental, 53, 25-38.

Consolo, M. (2017). El uso del término "dignidad" en el debate parlamentario de la Cámara de Senadores para la reforma, actualización y unificación de los Códigos Civil y Comercial de la Nación. Tras una búsqueda del sustrato filosófico del artículo 51. En R. RabbiBaldi (Coord.), Los derechos de la persona en el nuevo Código Civil y Comercial: aproximación a algunos aspectos novedosos (pp. 15-37). Buenos Aires, Argentina: La Ley.

Constitución Española. (1978). Recuperado de https://www.boe.es/legislacion/documentos/ConstitucionCASTELLANO.pdf

Convención Americana sobre Derechos Humanos. (1969). Recuperado de https://www.oas.org/dil/esp/tratados_b32_convencion_americana_sobre_derechos_humanos.htm

D’Agostino, F. (2002). La dignidad humana, tema bioético. En A. M. González, E. Postigo, y S. Aulestiarte (Eds.), Vivir y morir con dignidad (pp. 23-27). Pamplona, España: Eunsa.

Declaración Universal de Derechos Humanos. (1948). Recuperado de http://www.ohchr.org/EN/UDHR/Documents/UDHR_Translations/spn.pdf

Dupré, C. (2013). Human Dignity in Europe: A Foundational Constitutional Principle. European Public Law, 19(2), 319-340. 
Dworkin, R. (2006). Is Democracy Possible Here? Princeton, NJ: University Press.

Esain, J. A. (2017). El estado ambiental de derecho. Revista de Derecho Ambiental, 50, 23-49.

Esain, J. A. (2018). Fallo por el río Atuel. Federalismo Ambiental y derecho ambiental de aguas. La Ley, Tomo 2018-B, 113-127.

Esain, J. A. (2019). La Corte y la Ley de Glaciares bajo el escrutinio del control de complementariedad. La Ley. Tomo 2019-B, 389-404.

Falbo, A. (2019a). Jurisprudencia conceptual de derecho ambiental. Revista de Derecho Ambiental, 58, 1-9.

Falbo, A. (2019b) La Corte nacional y la emersión ambiental de los recursos naturales. La Ley, Tomo 2019-D, 383-389.

Gaylin, W. (1984). In Defense of the Dignity of Being Human. Hasting Center Report, 14(4), $18-22$.

Glare, P. (Ed). (1968). Oxford Latin Dictionary. Oxford, Reino Unido: Clarendon Press.

González Pérez, J. (1986). La dignidad de la persona. Madrid, España: Cívitas.

Hervada, J. (2000). Lecciones propedéuticas de Filosofía del Derecho. Pamplona, España: Eunsa.

Kant, I. (2008). Fundamentación para una metafísica de las costumbres. Madrid, España: Alianza.

Kant, I. (2016). Naturrecht Feyerabend [La "Introducción" a las Lecciones sobre derecho natural de Kant anotadas por Feyerabend] (M. Marey y N. Sánchez Madrid, Trads.). Con-Textos Kantianos. International Journal of Philosophy, 3, 391-414.

Kateb, G. (2011). Human Dignity. Cambridge, MA: Harvard University Press.

Kawall, J. (2017). A History of Environmental Ethics. En S. Gardiner, y A. Thompson, The Oxford Handbook of Environmental Ethics (pp. 12-23). Oxford, Reino Unido: University Press.

Laise, L. D. (2017). La dignidad de la persona y los derechos de incidencia colectiva como fundamento del derecho al acceso al agua potable en el Nuevo Código Civil y Comercial de la Nación Argentina. En R. Rabbi-Baldi (Coord.), Los derechos de la persona en el nuevo Código Civil y Comercial: aproximación a algunos aspectos novedosos (pp. 119-132). Buenos Aires, Argentina: La Ley.

Ley Fundamental de Alemania. (1949). Recuperado de https://www.btgbestellservice.de/pdf/80206000.pdf

Liddell, H., y Scott, R. (Eds). (1996). A Greek-English Lexicon. Oxford, Reino Unido: Clarendon Press.

López Alfonsín, M. (2012). Derecho ambiental. Buenos Aires, Argentina: Astrea. 
Lorenzetti, P. (2015). Compatibilización entre la esfera pública y la privada y entre el ámbito colectivo y el individual, en el Código Civil y Comercial de la Nación. Jurisprudencia Argentina, Tomo 2015-III, 14, 3-24.

Lubertino Beltrán, M. (2018). Los principios del Estado ambiental de Derecho en la Argentina. Revista de Derecho Ambiental, 53, 49-62.

Magnavacca, S. (2014). Léxico técnico de filosofía medieval. Buenos Aires, Argentina: Miño y Dávila.

Massini Correas, C. I. (1994). Los derechos humanos en el pensamiento actual. Buenos Aires, Argentina: Abeledo-Perrot.

Massini Correas, C. I. (2017). Sobre dignidad humana y derecho: la noción de dignidad de la persona y su relevancia constitutiva en el derecho. Prudentia Iuris, 83, 49-72.

Mattson, D., y Clark, S. (2011). Human dignity in concept and practice. Policy Sciences, 44(4), 303-319.

Melendo, T., y Millán-Puelles, L. (1996). Dignidad: ¿una palabra vacía? Pamplona, España: Eunsa.

Michael, L. (2014). Defining Dignity and Its Place in Human Rights. The New Bioethics, 20(1), 12-34.

Millán-Puelles, A. (2014). Sobre el hombre y la Sociedad. Madrid, España: Rialp.

Nino, C. (1989). Ética y derechos humanos. Buenos Aires, Argentina: Astrea.

Ober, J. (2012). Democracy's Dignity. The American Political Science Review, 106(4), 827846.

Pacto Internacional de Derechos Civiles y Políticos. (1966). Recuperado de https://www.ohchr.org/SP/ProfessionalInterest/Pages/CCPR.aspx

Pacto Internacional de Derechos Económicos, Sociales y Culturales. (1966). Recuperado de https://www.ohchr.org/sp/professionalinterest/pages/cescr.aspx

Pellegrini, N. (2018). El rol de la Corte y sus efectos en el Estado Ambiental de Derecho. Revista de Derecho Ambiental, 53, 63-72.

Pinto, M., y Liber, M. (2015). El derecho humano al agua en la jurisprudencia latinoamericana y de la Corte Suprema de Argentina. Naturaleza colectiva y exigibilidad inmediata del contenido mínimo. Revista de Derecho Ambiental, 42, 179-198.

Pritchard, M. (1972). Human Dignity and Justice. Ethics, 82(4), 299-313.

Quintana, E. (2017). Dignidad y deberes humanos. Prudentia Iuris, 83, 73-94.

Quiroga Lavié, H. (1996). El estado ecológico de derecho en la Constitución Nacional. La Ley, Tomo 1996-B, 950-960.

Rodríguez, C. A. (2019). Los humedales y su protección en un fallo de la Corte Suprema de Justicia. La Ley, Tomo 2019-D, 468-469. 
Sabsay, D., y Fernández, C. (2019). Ley de Glaciares y control de constitucionalidad: los derechos ambientales tomados en serio. Jurisprudencia Argentina, Tomo 2019-III, 409417.

Spaemann, R. (1988). Sobre el concepto de dignidad humana. Persona y Derecho, 19, 13-33.

Tugendhat, E. (1992). Ser-Verdad-Acción. Barcelona, España: Gedisa.

\section{Jurisprudencia}

“Aquino, Isacio c/Cargo Servicios Industriales S.A.”, Corte Suprema de Justicia de la Nación (21 de septiembre de 2004).

"Asociación Argentina de Abogados Ambientalistas de la Patagonia c/Santa Cruz, Provincia de y otro", Corte Suprema de Justicia de la Nación (26 de abril de 2016 y 21 de diciembre de 2016).

"Barrick Exploraciones Argentinas S.A. y otro c/Estado Nacional", Corte Suprema de Justicia de la Nación (4 de junio de 2019).

"Cruz, Felipa y otros c/Minería Alumbrera Limited y otro", Corte Suprema de Justicia de la Nación (23 de febrero de 2016).

"Fundación Ciudadanos Independientes c/San Juan, Provincia de, Estado Nacional y otros", Corte Suprema de Justicia de la Nación (20 de septiembre de 2016).

"Kersich, Juan Gabriel y otros c/Aguas Bonaerenses S.A.”, Corte Suprema de Justicia de la Nación (2 de diciembre de 2014).

"La Pampa, Provincia de c/Mendoza, Provincia de", Corte Suprema de Justicia de la Nación (1 de diciembre de 2017).

"Majul, Julio Jesús c/Municipalidad de Pueblo General Belgrano y otros", Corte Suprema de Justicia de la Nación (11 de julio de 2019).

"Mamani, Agustín Pio y otros c/Estado Provincial -Dirección Provincial de Políticas Ambientales y Recursos Naturales- y la Empresa Cram S.A.”, Corte Suprema de Justicia de la Nación (5 de septiembre de 2017).

"Martínez, Sergio Raúl c/Agua Rica LLc Suc. Argentina y su propietaria Yamana Gold Inc. y otro", Corte Suprema de Justicia de la Nación (2 de marzo de 2016).

"Mendoza Beatriz Silva y otros c/Estado Nacional", Corte Suprema de Justicia de la Nación (20 de junio de 2006, 22 de agosto de 2007, 8 de julio de 2008, 19 de febrero de 2015 y 12 de abril de 2018).

"Salas, Dino y otros c/Salta, Provincia de y Estado Nacional", Corte Suprema de Justicia de la Nación (29 de diciembre de 2008 y 26 de marzo de 2009). 
Este trabajo se enmarca en el Proyecto de Investigación en Derecho (DeCyT, DCT1822, años 2018-2020) "Naturaleza y límites de la interpretación jurídica: desafios en el Estado Constitucional de Derecho", acreditado y financiado por la Facultad de Derecho de la Universidad de Buenos Aires.

\section{Contribución autoral}

a) Concepción y diseño del trabajo; b) Adquisición de datos; c) Análisis e interpretación de datos;

d) Redacción del manuscrito; e) revisión crítica del manuscrito.

E. M. ha contribuido en a, b, c, d, e.

\section{Editor científico responsable}

Dra. María Paula Garat 